\title{
ROBUST ADAPTIVE LOMB PERIODOGRAM FOR TIME-FREQUENCY ANALYSIS OF SIGNALS WITH SINUSOIDAL AND TRANSIENT COMPONENTS
}

\author{
Zhiguo Zhang and Shing-Chow Chan \\ Department of Electrical and Electronic Engineering, The University of Hong Kong \\ Pokfulam Road, Hong Kong.
}

\begin{abstract}
This article introduces a robust adaptive Lomb periodogram (RALP) for time-frequency (TF) analysis of time series with sinusoidal and transient components, which are possibly non-uniformly sampled. It extends the conventional Lomb spectrum by windowing the observation data and adaptively selects the window lengths by the intersection of confidence intervals (ICI) rule. The influence of transient components to conventional time-frequency representation can be moderated using M-estimation of robust statistics. Instead of treating the transient components as impulsive noise and removing them, the proposed RALP TF distribution yields separately a time domain representation of the transient components and a conventional TF representation of the sinusoidal components, which greatly improves the visualization and detection of these components. Simulation results show that the proposed RALP differentiates the two kinds of components well, and offers better time and frequency resolutions than the conventional Lomb periodogram.
\end{abstract}

\section{INTRODUCTION}

Time-frequency distributions are useful tools for timefrequency analysis and visualization of signals. Two fundamental problems in time-frequency representations are: 1) the bias and variance trade-off problem and 2) the suppression of the adverse effects of transient components such as outliers.

The first problem is concerned with the choice of window length in the TF distribution. For slowly varying sinusoidal components, a long time window is desirable because more samples can be used to determine their amplitudes. The reduced variances of the spectral coefficients give a better frequency resolution at the expense of a lower time resolution. On the contrary, for fast varying components, a small window is preferred in order to reduce the bias in estimation. As a result, a better time resolution is achieved for tracking these fast varying components at the cost of a lower frequency resolution. The first problem is usually addressed by adaptively choosing the window length using the intersection of confidence intervals (ICI) rule. Katkovnik et al [5] developed such algorithms and had successfully applied it to Wigner distribution [6] and Mperiodogram [7].

The second problem was first observed and addressed in [4], where a robust M-periodogram using M-estimation was proposed to combat the adverse effect of outliers in the conventional periodogram. The basic idea is to employ a nonquadratic residual loss function when fitting the sinusoids to the observations, instead of using the square function in the conventional method. The latter is known to have a very high sensitive to outliers. A robust Wigner distribution based on a similar concept was proposed in [3]. One important limitation of this approach is that transient components are treated as outliers and are suppressed. However, in many applications, transient components might represent useful information and they should not be removed entirely.

In this paper, we will employ the Lomb periodogram as our basic time-frequency representation $[1,2]$. Its basic idea is to estimate the amplitude of a given sinusoid with a certain frequency $\omega$ by least squares fitting the sinusoids to the observed non-uniform data points. An important advantage of the Lomb periodogram is that it can be applied to non-uniformly spaced data. One significant drawback of the conventional Lomb periodogram is that it estimates the sinusoidal components using the entire data set, which is unsuitable in time-varying environment. To over this problem, a windowed version of the Lomb periodogram was proposed recently in [9] by the authors. Moreover, the ICI rule is employed to select optimal windows in the time-frequency domain. Simulation results showed that better bias-variance trade-off could be achieved as compared with the conventional Lomb periodogram. Here, we extend the work in [9] to address the second problem mentioned above by introducing M-estimation to the adaptive Lomb periodogram. This is motivated by the M-periodogram in [4] for uniform data with two important differences, apart from being able to handle non-uniform spaced observations. First of all, the proposed RALP employs time-frequency adaptive smoothing in order to achieve a better bias-variance trade-off. Secondly, the transient components are not treated simply as noise and removed. Instead, they are suppressed using M-estimation so that we can obtain an ALP for the sinusoidal components alone. By recovering the sinusoidal components, the transients in the time domain can be isolated, giving a TF- and a time-domain representation, respectively, for the sinusoidal and transient contents of the non-uniform spaced observations.

The paper is organized as follows: in Section 2, the robust windowed Lomb periodogram is proposed. The adaptive Lomb periodogram and window selection are introduced in Section 3. Section 4 is devoted to the estimation of the sinusoidal and transient components using the robust adaptive Lomb spectrum. Simulation results and comparisons are described in Section 5. Finally, conclusions are drawn in Section 6.

\section{ROBUST WINDOWED LOMB PERIODOGRAM}

Given a set of $N$ non-uniform discrete-time samples $x_{t}$ of a signal $m(t)$. The Lomb periodogram $P(\omega)$ at a frequency $\omega$ is computed from the observations using a least-squares fit of $x_{t}$ by sinusoids with the given frequency:

$$
x_{t}=c_{1}(\omega) \cdot \cos (\omega t)+c_{2}(\omega) \cdot \sin (\omega t)+e_{t},
$$


where the additional components $e_{t}$ is assumed to be an independent and identically distributed (i.i.d.) Gaussian white noise $[4,8]$. The parameter vector $C(\omega)=\left[c_{1}(\omega), c_{2}(\omega)\right]^{\prime}$ can be solved by minimizing the quadratic loss function: $\hat{C}(\omega)=\arg \min _{C} \sum_{t}\left|e_{t}\right|^{2}$, which yields a least-squares problem.

The power spectrum can then be computed from the estimation of $C(\omega)$ as $P(\omega)=\|\hat{C}(\omega)\|^{2}$. By taking into account the time shift $\tau$ of the sinusoids, Lomb [1] and Scargle [2] suggested the following definition of Lomb periodogram:

$$
\begin{aligned}
& P(\omega)=\frac{1}{2 \sigma^{2}}\left\{\frac{\left[\sum_{t}\left(x_{t}-\bar{x}_{t}\right) \cdot \cos (\omega(t-\tau))\right]^{2}}{\sum_{t} \cos ^{2}(\omega(t-\tau))}\right. \\
&\left.+\frac{\left[\sum_{t}\left(x_{t}-\bar{x}_{t}\right) \cdot \sin (\omega(t-\tau))\right]^{2}}{\sum_{t} \sin ^{2}(\omega(t-\tau))}\right\}
\end{aligned},
$$

where $\bar{x}_{t}=\left(\sum_{t} x_{t}\right) / N$ is introduced to make the observations zero mean, and $\sigma^{2}=\sum_{t}\left(x_{t}-\bar{x}_{t}\right)^{2} /(N-1)$ is a normalization constant. The constant $\tau$ is given by $\tan (2 \omega \tau)=\sum_{t} \sin (2 \omega t) /\left\{\sum_{t} \cos (2 \omega t)\right\}$.

In order to track time-varying signals and produce a timefrequency Lomb spectrum, a window $w(t)$, which is centered at time $t$, is usually introduced [9] so that the Lomb periodogram of the windowed data $x_{t, w}=x_{t} w_{t}$ at a given time instant $t$ is computed. It gives a windowed Lomb periodogram (or more precisely, a TF distribution) $P(t, \omega)$ of the signal at different time instants $t$. This yields:

$$
\begin{array}{r}
P(t, \omega)=\frac{1}{2 \sigma_{t}^{2}}\left\{\frac{\left[\sum_{w}\left(x_{t, w}-\bar{x}_{t, w}\right) \cdot \cos \omega\left(t_{w}-\tau_{t}\right)\right]^{2}}{\sum_{w} \cos ^{2} \omega\left(t_{w}-\tau_{t}\right)}\right. \\
\left.+\frac{\left[\sum_{w}\left(x_{t, w}-\bar{x}_{t, w}\right) \cdot \sin \omega\left(t_{w}-\tau_{t}\right)\right]^{2}}{\sum_{w} \sin ^{2} \omega\left(t_{w}-\tau_{t}\right)}\right\},
\end{array},
$$

where $\bar{x}_{t, w}$ and $\sigma_{t}$ are respectively the mean value and the standard deviation of the data in the window $w_{t}$, and $\tau_{t}$ is the offset in the window $w_{t}$ at time $t$.

Due to the use of least-squares criterion in the fitting process, transient components, which appear as outliers to the model, will significantly affect the periodogram (to be illustrated later in the simulation section). To moderate this adverse effect of the transient components, the M-estimation is employed where the loss function is modified to $\hat{C}_{\rho}(\omega)=\arg \min _{C} \sum_{t} \rho\left(e_{t}\right)$, and $\rho(\cdot)$ is an M-estimate function. Here, we use the traditional Huber function to de-emphasize the "outliers":

$$
\rho(e)=\left\{\begin{array}{cc}
e^{2} / 2, & 0<|e|<\xi \\
\xi|e|-\xi^{2} / 2 & |e| \geq \xi
\end{array},\right.
$$

where $\xi$ is a threshold used to "reject the outliers". Other functions such as the Hampel three parts red-descending function can also be used. By setting the derivatives of $E_{\rho}=\sum_{t} \rho\left(e_{t}\right)$ with respect to $\hat{C}(\omega)$ to be zero, we obtain the following recursive algorithm for computing the robust Lomb periodogram based on Iterated Weighted Least Squares (IWLS):
1. Calculate a starting value for $\hat{C}_{0}(\omega)$ using least-squares algorithm [8]:

$$
\hat{C}_{0}(\omega)=\left(\begin{array}{c}
\frac{\sum_{t}\left(x_{t}-\bar{x}_{t}\right) \cdot \cos (\omega(t-\tau))}{\sum_{t} \cos ^{2}(\omega(t-\tau))} \\
\frac{\sum_{t}\left(x_{t}-\bar{x}_{t}\right) \cdot \sin (\omega(t-\tau))}{\sum_{t} \sin ^{2}(\omega(t-\tau))}
\end{array}\right) .
$$

2. At the $k$-th iteration, compute the estimation error of the least-squares fitting:

$$
e_{k}(\omega, t)=\left(x_{t}-\bar{x}_{t}\right)-\hat{C}_{k-1}(\omega)^{T} \cdot\left(\begin{array}{c}
\cos (\omega(t-\tau)) \\
\sin (\omega(t-\tau))
\end{array}\right) .
$$

3. Calculate the updated estimate of $\hat{C}_{k}(\omega)$ by a weighted least-squares:

$$
\hat{C}_{k}(\omega)=\left(\begin{array}{c}
\frac{\sum_{t} q_{k}(\omega, t) \cdot\left(x_{t}-\bar{x}_{t}\right) \cdot \cos (\omega(t-\tau))}{\sum_{t} \cos ^{2}(\omega(t-\tau))} \\
\frac{\sum_{t} q_{k}(\omega, t) \cdot\left(x_{t}-\bar{x}_{t}\right) \cdot \sin (\omega(t-\tau))}{\sum_{t} \sin ^{2}(\omega(t-\tau))}
\end{array}\right),
$$

where $q_{k}(\omega, t)=\left(\Psi\left(e_{k}(\omega, t)\right) / e_{k}(\omega, t)\right)$ is the weighting function, and

$$
\Psi\left(e_{k}\right)=\rho^{\prime}\left(e_{k}\right)=\left\{\begin{array}{cc}
e_{k}, & 0<\left|e_{k}\right|<\xi_{k} \\
\operatorname{sgn}\left(e_{k}\right) \cdot \xi & \left|e_{k}\right| \geq \xi_{k}
\end{array} .\right.
$$

The threshold $\xi$ in (8) is used to restrain the outliers (transient components) detected from the estimation error $e_{k}$, and it can be adaptively updated as $\xi_{k}=\lambda \cdot \sigma_{e_{k}}$, where $\sigma_{e_{k}}$ is the standard deviation of the "impulse-free" estimation error $e_{k}$ [10]. The parameter $\lambda$ can be chosen as 1.2817, $1.96,2.576$, or 3.091 according to the intensity of outliers. So we can see that when there is a large error $e_{k}(\omega, t)$ at time $t$, the data $x_{t}-\bar{x}_{t}$ will be given a small weight at the next least-squares iteration to moderate the adverse influence of outliers (transient components) at time $t$.

4. Use the updated $\hat{C}_{k}(\omega)$ to calculate the estimation error $e_{k+1}(\omega, t)$ and repeat the process until converge.

5. The robust Lomb periodogram $\hat{C}_{k}(\omega)=\left(\begin{array}{c}\hat{c}_{k, 1}(\omega) \\ \hat{c}_{k, 2}(\omega)\end{array}\right)$ is $[1,8]$ :

$$
P_{k}(\omega)=\frac{1}{2} \|\left(\begin{array}{l}
\left.\hat{c}_{k, 1}(\omega) \cdot \sqrt{\sum_{t} \cos ^{2}(\omega(t-\tau))}\right) \|^{2} \\
\left.\hat{c}_{k, 2}(\omega) \cdot \sqrt{\sum_{t} \sin ^{2}(\omega(t-\tau))}\right)
\end{array} .\right.
$$

Simulation results showed that the algorithm usually converges in $k=3 \sim 5$ iterations and never exceeded 10 .

\section{ADAPTIVE WINDOW SELECTION}

The selection of window length is crucial to the timefrequency resolution of the Lomb $\mathrm{TF}$ distribution $P(t, \omega)$. Motivated by the novel bandwidth selection rule in $[5,6,7]$, we propose to use the ICI rule for determining the window lengths of the adaptive Lomb periodogram in the time-frequency domain. Suppose that we are given a set of finite window sizes in ascending order of magnitude: $H=\left\{h_{k} \mid h_{1}<h_{2}<\ldots<h_{K}\right\}$. For 
each window size $h_{k}$, we will obtain a Lomb periodogram $P\left(t, \omega ; h_{k}\right)$. The variance, $\operatorname{Var}(\cdot)$, and the bias, $\operatorname{bias}(\cdot)$, of these Lomb periodograms are functions of the time window $h_{k}$, so is the mean square error ( $M S E$ ), which is given by:

$$
\operatorname{MSE}\left(t, \omega ; h_{k}\right)=\operatorname{Var}\left[P\left(t, \omega ; h_{k}\right)\right]+\operatorname{bias}^{2}\left[P\left(t, \omega ; h_{k}\right)\right] \text {. (10) }
$$

The variance and bias are respectively decreasing and increasing functions of the window size $h_{k}$. Therefore, there exists an optimal window such that the two terms are equal and $\operatorname{MSE}\left(t, \omega ; h_{k}\right)$ is minimized. The ICI rule will examine a sequence of confidence intervals of the estimates $P\left(t, \omega ; h_{k}\right)$ to determine the optimal window lengths. By using an adaptive window for each sinusoid and at each time instant $t$, better bias and variance trade-off can be achieved. The optimal window size array of the whole time-frequency domain is $h_{k^{+}}(t, \omega)$ and the optimal Lomb periodogram is $P\left(t, \omega ; h_{k^{+}}\right)$. More detail of the algorithm can be found in [9]. For more information of the ICI rule, see $[5,6,7]$. Combining the adaptive window selection method with robust periodogram in Section 2, we will get the final robust adaptive Lomb periodogram (RALP).

\section{SINUSOIDAL AND TRANSIENT COMPONENTS ESTIMATION}

Lomb periodogram was designed to compute the power spectrum. Hence, the phase information, which is essential to the reconstruction of the time series, is discarded. To estimate the transient components, however, we need to find the phase information to reconstruct the sinusoidal components from the robust windowed Lomb spectrum. Although we can recover the data from $\hat{C}(\omega)$ in (7), we still want to use some fast algorithm, such as the Inverse Fast Fourier transform (IFFT). To this end, we define from (2) the Lomb spectrum as follows:

$$
\begin{aligned}
X_{\text {Lomb }}(\omega)=\frac{1}{\sqrt{2}} & \left\{\frac{\sum_{t}\left(x_{t}-\bar{x}_{t}\right) \cdot \cos (\omega t)}{\sqrt{\sum_{t} \cos ^{2} \omega t}},\right. \\
& \left.-j \cdot \frac{\sum_{t}\left(x_{t}-\bar{x}_{t}\right) \cdot \sin (\omega t)}{\sqrt{\sum_{t} \sin ^{2} \omega t}}\right\}
\end{aligned}
$$

where $j=\sqrt{-1}$. Mathias et al. [8] had proved that the time shift $\tau$ contains the phase information and does not affect the amplitudes. So, we have $P(\omega)=\frac{1}{\sigma^{2}}\left\|X_{\text {Lomb }}(\omega)\right\|^{2}$, where $\sigma^{2}$ is the variance of observation used as a normalization factor. Because $\sum_{t} \cos ^{2}(\omega t) \approx N / 2, \sum_{t} \sin ^{2}(\omega t) \approx N / 2$ [1], we have:

$$
X_{\text {Lomb }}(\omega) \approx \frac{\sum\left(x_{t}-\bar{x}_{t}\right) \cdot \cos (\omega t)-j \cdot \sum\left(x_{t}-\bar{x}_{t}\right) \cdot \sin (\omega t)}{\sqrt{N}},
$$

where $\sum_{t}\left(x_{t}-\bar{x}_{t}\right) \cdot \cos (\omega t)-j \cdot \sum_{t}\left(x_{t}-\bar{x}_{t}\right) \cdot \sin (\omega t)$ has the physical meaning of Discrete-Time Fourier transform of $\left(x_{t}-\bar{x}_{t}\right)$. So, by applying IFFT to $\sqrt{N} \cdot X_{\text {Lomb }}(\omega)$, the original observation can be reconstructed approximately. The same idea can be applied to the robust adaptive Lomb periodogram/spectrum in Section 3, only the sinusoidal components are recovered and the transient components can be detected from the difference between the reconstructed and the observed data. Thus, a TF- and a time-domain representations, respectively, for the sinusoidal and transient components of the non-uniform spaced observations are obtained.

\section{SIMULATION RESULTS}

\section{Example 1: Uniformly Sampled Data}

A sinusoidal component $m(t)$ with an instantaneous frequency of $\omega(t)=0.08 \pi \cdot \arcsin (t-50)+0.5 \pi$ is considered. The observed data $x_{t}$ are uniformly sampled at $t=1,2 \cdots 100$, and are assumed to be corrupted by additive Gaussian noise with a SNR of $20 \mathrm{~dB}$. Two transient components with variance 200 are added at $t=30$ and $t=80$ for the sake of visualization. The windows lengths considered are 17, 33, 65, and 129. Fig.1 shows the comparison of conventional windowed Lomb periodogram (based on least-squares) and robust windowed Lomb periodogram (based on M-estimation) with fixed window lengths. We can see that when the window length is small, the time resolution is high but the frequency resolution is low. As a result, the transient components have a larger effect when the window is small. Robust M-estimation helps to moderate the adverse effect of the transient components, but the timefrequency resolution is limited by the fixed window size. Figure 2 shows the results for the proposed RALP and the ALP in [9]. The time-frequency resolution is significantly improved over the fixed window case. Further, the adverse effect of the transient components is effectively suppressed in the RALP. Fig.3 shows the reconstructed results for the RALP. The transient components, which are obtained from the difference of the original and the reconstructed data, are satisfactorily isolated, illustrating the effectiveness of the proposed algorithm.

\section{Example 2: Non-uniformly Sampled Data}

We now consider a sinusoidal signal $m(t)$ with an instantaneous frequency $\omega(t)=0.04 \pi \cdot \arcsin (t-50)+\pi . \quad x_{t}$ are obtained from 100 non-uniform samples of $m(t)$ randomly chosen between time 1 100 with the same additive noise as example 1. Other parameter settings are the same as example 1. Fig.4 shows the results for the RALP. Compared with Example 1 (uniform sampled data), we notice the following:

1. Because the average time interval is 1 in $x_{t}$, the Nyquist frequency should be 0.5 . However, because of non-uniform sampling, the Lomb periodogram can detect frequency components larger than the Nyquist frequency 0.5.

2. The non-uniform case has larger noise variance and more "frequency leakage". Orientation analysis and directional smoothing are effective to remove these undesirable components [9].

\section{CONCLUSION}

A new RALP spectrum for time-frequency analysis of time series with both sinusoidal and transient components is presented in this paper. The conventional Lomb spectrum is extended by windowing the observation data and the window lengths are adaptively selected by the ICI rule. Transient components are moderated using M-estimation of robust statistics to restrain their influence to time-frequency representation of sinusoidal components. Experiment results 
show that our proposed RALP time-frequency distribution can yield separately a time-domain representation of the transient components and a time-frequency representation of the sinusoidal components, which offers better time and frequency resolutions than the conventional Lomb periodogram.

\section{REFERENCES}

[1] N. R. Lomb, "Least-squares frequency analysis of unequally spaced data," Astrophys. Space Sci., Vol. 39, pp. 447-462, 1976.

[2] J. D. Scargle, "Studies in astronomical time series analysis II: Statistical aspects of spectral analysis of unevenly sampled data," Astrophysical Journal, Vol. 263, pp. 835-853, 1982.

[3] I. Djurovic and L. Stankovic, "Robust Wigner Distribution With Application to the Instantaneous Frequency Estimation," IEEE Trans. Sig. Proc., Vol. 49, No. 12, pp. 2985-2993, 2001.

[4] V. Katkovnik, "Robust M-periodogram," IEEE Trans. Sig. Proc., Vol.46, No.11, pp. 3104-3109, 1998.

[5] V. Katkovnik and L. Stankovic, "Periodogram with varying and data-driven window length," Signal Processing, Vol. 67, No. 3, pp. 345-358, 1998.

[6] V. Katkovnik and L. Stankovic, "Instantaneous Frequency Estimation Using the Wigner Distribution with Varying and Data-Driven Window Length," IEEE Trans. Sig. Proc., Vol. 46, No. 9, pp. 2315-2325, 1998.

[7] I. Djurovic, V. Katkovnik and L. Stankovic, "Instantaneous frequency estimation based on the robust spectrogram," Proc. IEEE ICASSP'01, Vol. 6, pp. 3517-3520, 2001.

[8] A. Mathias, F. Grond, R. Guardans, D. Seese, M. Canela and H.H. Diebner, "Algorithms for Spectral Analysis of Irregularly Sampled Time Series," Journal of Statistical Software, Vol. 11, Issue 2, pp. 1-30, 2004.

[9] S. C. Chan and Z. G. Zhang, "Adaptive Window Selection and Smoothing of Lomb Periodogram for Time-Frequency Analysis of Time Series," Proc. IEEE 2004 Midwest Symposium on Circuits and Systems, Vol. II, pp. 137-140, July 25-28, 2004.

[10] S. C. Chan and Z. G. Zhang, "Multi-resolution Analysis of Non-uniform Data with Jump Discontinuities and Impulsive Noise Using Robust Local Polynomial Regression," IEEE ICASSP'04, Canada, vol. II, pp. 769-772, May 17-21, 2004.

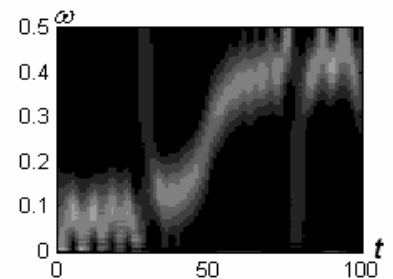

(a)

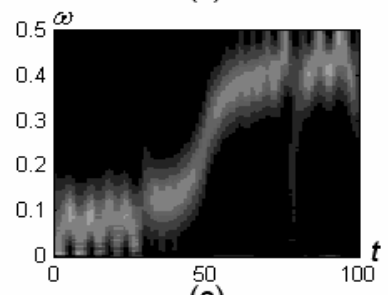

(c)

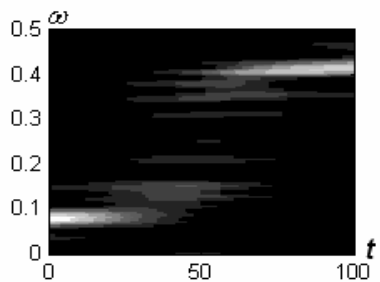

(b)

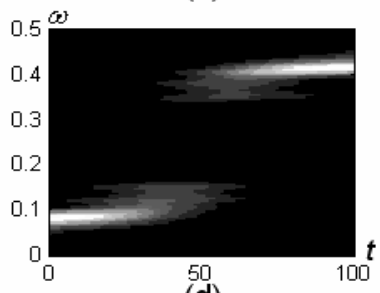

(d)
Fig. 1: conventional windowed Lomb periodogram with fixed window lengths (a) $h=17$, (b) $h=129$;

robust windowed Lomb periodogram with fixed window lengths (c) $h=17$, (d) $h=129$.

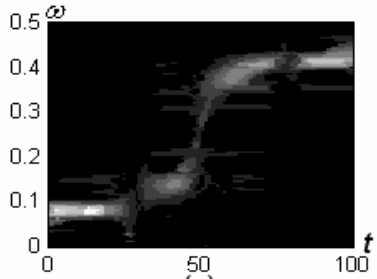

(a)

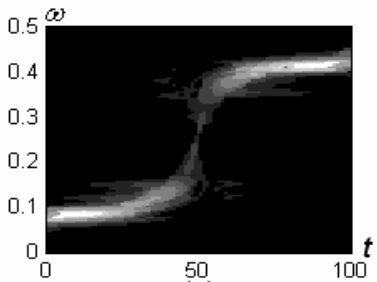

(c)

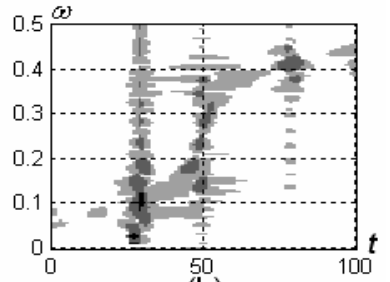

(b)

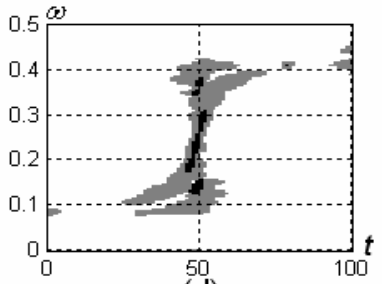

(d)
Fig. 2: (a) conventional adaptive Lomb periodogram, (b) conventional adaptive time window lengths, (c) robust adaptive Lomb periodogram, (d) robust adaptive time window lengths.

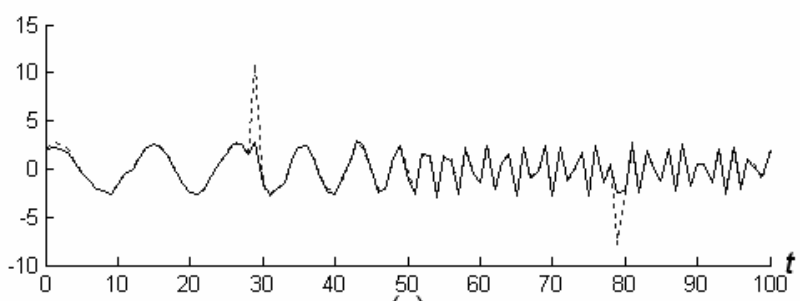

(a)

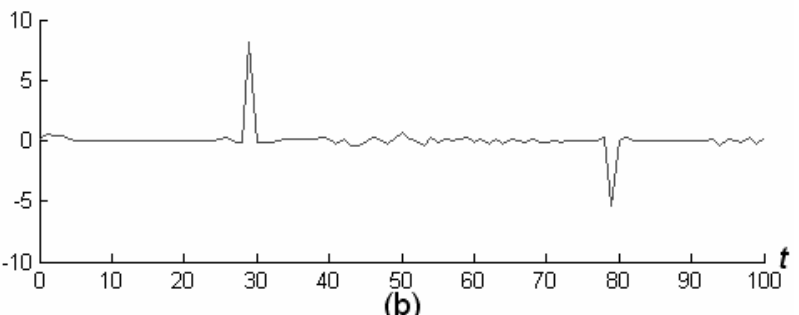

Fig. 3: (a) original uniform sampled observation data (dotted line) and reconstructed sinusoidal components from robust adaptive Lomb spectrum (solid line), (b) transient components detection.

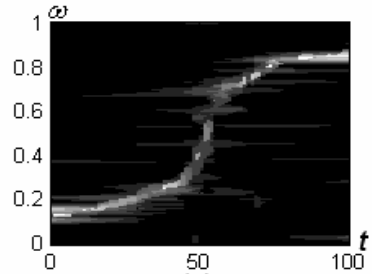

(a)

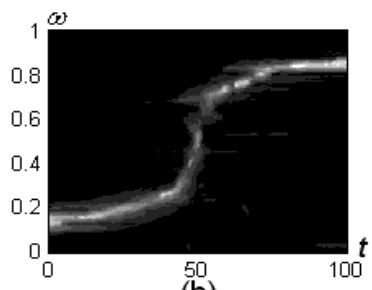

(b)
Fig. 4: (a) robust Lomb periodogram with adaptive time window, (non-uniform data), (b) robust Lomb periodogram after orientation analysis and directional smoothing. 